\title{
Síndrome da artéria mesentérica superior - Uma complicação incomum após correções cirúrgicas de deformidades da coluna
}

\section{Superior Mesenteric Artery Syndrome - An Uncommon Complication After Surgical Corrections of Spinal Deformities}

\author{
Alex Oliveira de Araujo ${ }^{10}$ Rafael Garcia de Oliveira ${ }^{10}$ Amanda Jordão de Castro Arraes ${ }^{2}$ AD $^{\circ}$ \\ Eduardo Magalhães Mamare ${ }^{2(0)}$ Ivan Dias Rocha ${ }^{3(0)}$ Cicero Ricardo Gomes ${ }^{1(1)}$ \\ 1 Departamento de Ortopedia, Rede Sarah de Hospitais de \\ Reabilitação, Brasília, DF, Brasil \\ 2 Departamento de Cirurgia Geral, Rede Sarah de Hospitais de \\ Reabilitação, Brasília, DF, Brasil \\ Endereço para correspondência Alex Oliveira de Araujo, MD, Rede \\ Sarah de Hospitais de Reabilitação, SMHS 501, Bloco A, Brasília, \\ Distrito Federal, 70.335-901, Brasil \\ (e-mail: alexiotfmusp@gmail.com).
}

${ }^{3}$ Instituto de Ortopedia e Traumatologia, Hospital das Clínicas, Faculdade de Medicina, Universidade de São Paulo, São Paulo, SP, Brasil

Rev Bras Ortop 2021;56(4):523-527.

\begin{abstract}
Resumo
A compressão vascular da terceira parte do duodeno pela artéria mesentérica superior resulta no desenvolvimento de uma condição incomum de oclusão do trânsito duodenal conhecida como síndrome da artéria mesentérica superior. Este fenômeno pode acontecer após cirurgias de correção de deformidades da coluna, e sua taxa de ocorrência é de $\sim 0,5$ a 4,7\% dos casos. Isso ocorre em virtude da alteração do posicionamento do ponto de emergência da artéria, decorrente da mudança do comprimento do tronco após a cirurgia, e está associado a fatores de risco, como baixo índice de massa corpórea e perda ponderal. Os pacientes costumam se apresentar com um quadro de oclusão intestinal, com dor abdominal, náusea, vômito bilioso e saciedade precoce. O reconhecimento desta condição é importante para instituir o tratamento adequado, que varia do tratamento clínico, com sondagem e

Palavras-chave

- síndrome da artéria mesentérica superior/ complicações

- obstrução intestinal

- fusão vertebral

- escoliose/cirurgia descompressão gástrica associados a suporte nutricional; à necessidade de abordagem cirúrgica. Complicações secundárias relacionadas à síndrome da artéria mesentérica superior incluem: recuperação cirúrgica e nutricional retardadas, problemas com a cicatrização e hospitalização prolongada. O objetivo do presente estudo é relatar um caso de síndrome da artéria mesentérica superior, ocorrido em um paciente com escoliose neuromuscular secundária a sequela de mielite transversa, submetido ao tratamento cirúrgico da deformidade da coluna.
\end{abstract}

recebido

27 de Maio de 2020

aceito

16 de Setembro de 2020
DOI https://doi.org/

10.1055/s-0040-1722258. ISSN 0102-3616. (c) 2021. Sociedade Brasileira de Ortopedia e Traumatologia. All rights reserved.

This is an open access article published by Thieme under the terms of the Creative Commons Attribution-NonDerivative-NonCommercial-License, permitting copying and reproduction so long as the original work is given appropriate credit. Contents may not be used for commercial purposes, or adapted, remixed, transformed or built upon. (https://creativecommons.org/ licenses/by-nc-nd/4.0/)

Thieme Revinter Publicações Ltda., Rua do Matoso 170, Rio de Janeiro, RJ, CEP 20270-135, Brazil 


Abstract

Keywords
- superior mesenteric
artery syndrome/
complications
- intestinal obstruction
- spinal fusion
- scoliosis/surgery

Vascular compression of the third part of the duodenum by the superior mesenteric artery results in an unusual occlusion of the duodenal transit known as superior mesenteric artery syndrome. This syndrome can occur after surgeries to correct spinal deformities in a rate ranging from $0.5 \%$ to $4.7 \%$. It results from a positional alteration of the artery emergency point due to a change in trunk length after surgery. It is associated with risk factors such as low body mass index and weight loss. Patients usually present with intestinal occlusion, abdominal pain, nausea, bilious vomiting, and early satiety. Superior mesenteric artery syndrome must be recognized early to institute an adequate treatment, which can be clinical (with gastric tube for decompression and nutritional support) or require a surgical procedure. Secondary complications related to superior mesenteric artery syndrome include delayed surgical and nutritional recovery, healing problems, and prolonged hospitalization. The present study aims to report a case of superior mesenteric artery syndrome in a patient with neuromuscular scoliosis secondary to a transverse myelitis who underwent surgical treatment for spinal deformity correction.

\section{Introdução}

A compressão vascular da terceira parte do duodeno pela artéria mesentérica superior (AMS) resulta no desenvolvimento de uma condição incomum de oclusão do trânsito do duodeno conhecida como síndrome da artéria mesentérica superior (SAMS). A sua etiologia está ligada à relação anatômica da terceira parte do duodeno com o ângulo aortomesentérico. ${ }^{1}$ A obstrução é causada pela compressão extrínseca criada pelo feixe neurovascular mesentérico superior e a base da raiz do mesentério do intestino delgado, que se cruzam anteriormente ao duodeno. ${ }^{2}$

A AMS sai da aorta no nível do corpo vertebral de L1, está envolvida por um tecido adiposo e linfático e forma um ângulo de 45 a $60^{\circ}$ com a aorta. O duodeno geralmente cruza a aorta ao nível de L3 e é suspenso pelo ligamento de Treitz. $O$ desequilíbrio na relação entre essas estruturas pode levar à SAMS $^{3}$ (-Figura 1).

A incidência da SAMS após procedimentos cirúrgicos para correção de deformidades da coluna varia entre 0,5 e 4,7\%, e em geral afeta predominantemente pacientes adolescentes,

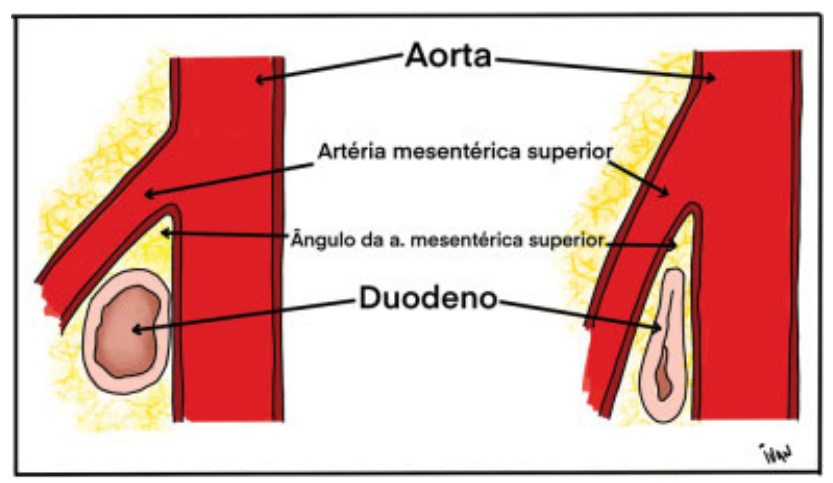

Fig. 1 Ilustração demonstrando a relação anatômica da $3^{\mathrm{a}}$ porção do duodeno com a artéria mesentérica superior e a sua obstrução observada na síndrome da artéria mesentérica superior. astênicos e altos. ${ }^{1}$ Acredita-se que estes pacientes tenham menos gordura retroperitoneal, perdendo o "coxim adiposo" entre o duodeno e a artéria mesentérica. ${ }^{4}$

A apresentação clínica da SAMS é caracterizada por dor abdominal, náusea, vômito bilioso e saciedade precoce, e o seu principal diagnóstico diferencial é o íleo paralítico. ${ }^{4}$ Complicações secundárias relacionadas à síndrome da artéria mesentérica superior incluem: recuperação cirúrgica e nutricional retardadas, problemas com a cicatrização e hospitalização prolongada. $^{5}$

O objetivo do presente estudo é relatar um caso de síndrome da artéria mesentérica superior, ocorrido em um paciente com escoliose neuromuscular submetido ao tratamento cirúrgico da deformidade da coluna.

\section{Relato de Caso}

Paciente do sexo masculino, admitido aos 12 anos de idade, portador de sequela de mielite transversa viral, com comprometimento diplégico sensitivo-motor.

Durante o seguimento, o paciente apresentou uma escoliose neuromuscular progressiva que necessitou de tratamento cirúrgico. Na ocasião, o paciente apresentava uma escoliose de $63^{\circ}$ (T2 a L2), associada a cifose na transição toracolombar de $37^{\circ}$ (T10 a L2). Na internação, apresentava-se com índice de massa corpórea (IMC) de 15,87 (magreza) e havia perdido $13 \mathrm{~kg}$ nos 2 anos precedentes (-Figuras 2 e 3 ).

Foi realizada artrodese posterior da coluna de T2 a S1, sem intercorrências, e o paciente evoluiu com melhora clínica progressiva nos primeiros dias de pós-operatório (DPO). No $9^{\circ} \mathrm{DPO}$, iniciou quadro de vômitos e febre. No $11^{\circ} \mathrm{DPO}$, manteve-se com vômitos recorrentes e apresentou distensão abdominal e inapetência. A tomografia de abdome mostrou uma grande distensão gástrica estendendo-se até a terceira porção do duodeno. Em virtude da região anatômica onde ocorreu a dificuldade de trânsito intestinal, foi considerada a possibilidade de SAMS (- Figura 4). 


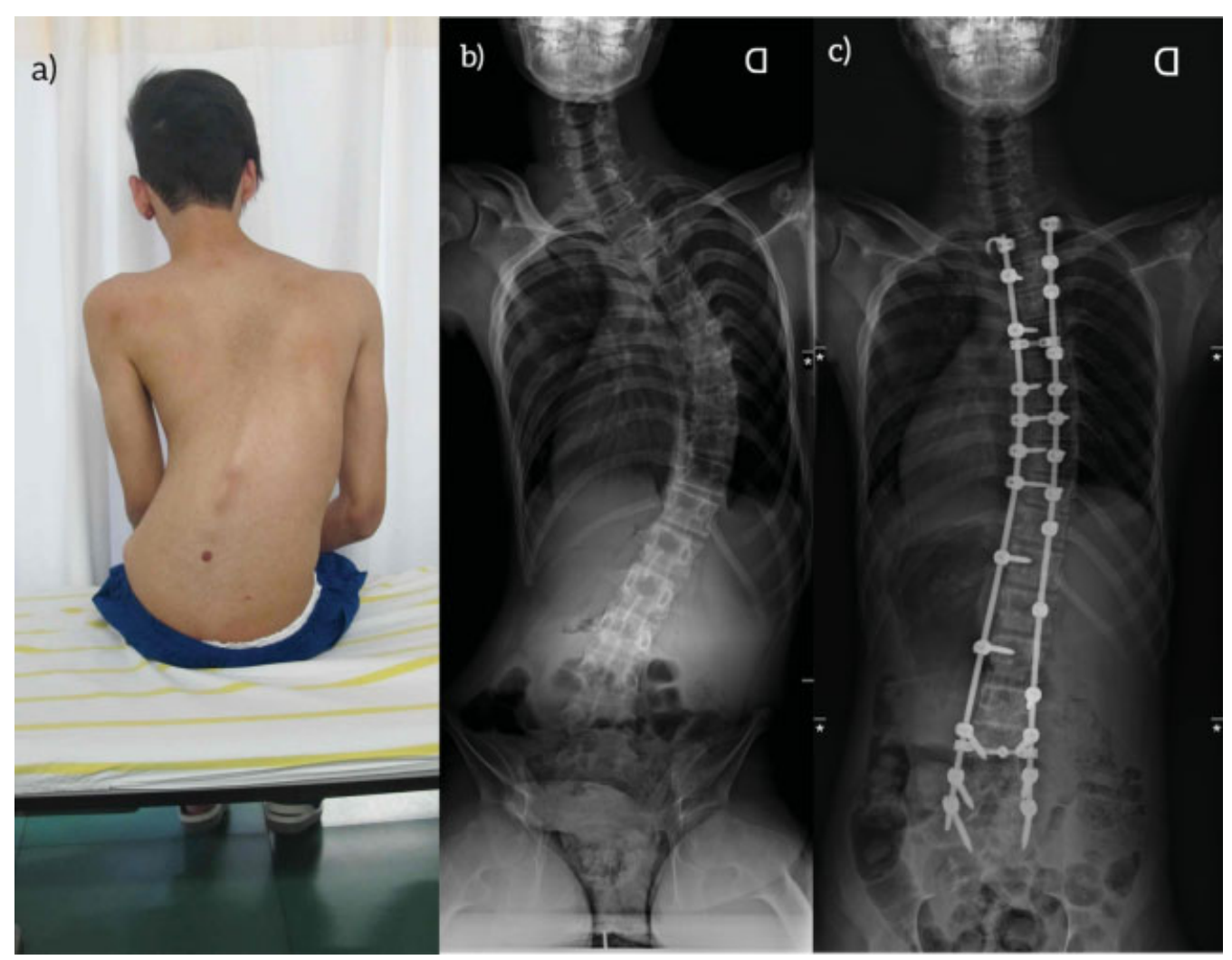

Fig. 2 (a) Imagem clínica pré-operatória (b) Radiografia panorâmica da coluna pré-operatória (c) Radiografia panorâmica pós-operatória.

Foi realizada sondagem nasogástrica com saída inicial de $1.200 \mathrm{~mL}$ de líquido com característica biliosa e alívio parcial dos sintomas; contudo, o paciente não apresentou melhora do quadro nos dias seguintes e foi indicada intervenção cirúrgica pela equipe de cirurgia geral. O paciente foi submetido a uma endoscopia digestiva alta, sendo observada grande quantidade de líquido de estase desde o esôfago até o duodeno, além de uma obstrução completa da luz duodenal na sua $3^{3}$ porção e a presença de pulsação atribuída à artéria mesentérica superior, que o comprimia. Foi realizada uma laparotomia mediana supra umbilical, sendo feita derivação duodenojejunal cerca de $20 \mathrm{~cm}$ após o ângulo de Treitz (-Figura 5).

Houve uma evolução satisfatória no pós-operatório. A ingestão de líquidos foi iniciada no $6^{\circ}$ dia pós-operatório, e a sonda nasogástrica foi retirada no $8^{\circ}$. No $11^{\circ}$ dia, foi iniciada alimentação oral líquida e realizada progressão lenta da dieta para alimentos pastosos e sólidos. Após 17 dias, o paciente recebeu alta hospitalar. No primeiro retorno, após 7 dias da alta, pesava $46,3 \mathrm{~kg}$. Após 4 meses, o seu peso era de $50 \mathrm{~kg}$, com ritmo intestinal normalizado e sem nenhuma intolerância alimentar. No $8^{\circ}$ mês, estava pesando $54 \mathrm{~kg}$, totalizando um ganho ponderal de $7,7 \mathrm{~kg}$, e não apresentava qualquer sintoma gastrointestinal.

\section{Discussão}

Náuseas e vômitos são frequentes em pacientes submetidos a artrodese da coluna para correção de escoliose. Podem decorrer do uso de medicações analgésicas como os opioides, ou mesmo de um quadro de íleo paralítico, comum após cirurgias de grande porte. Geralmente, o íleo paralítico é resolvido dentro dos primeiros 5 a 7 dias. $^{6}$

A combinação da correção cirúrgica da deformidade da coluna com a perda ponderal prévia aumenta o risco para a SAMS. Os sintomas da síndrome geralmente tornam-se aparentes após 6 a 8 dias da cirurgia e, diferente do íleo paralítico, os ruídos hidroaéreos costumam estar presentes. ${ }^{7}$ Existem casos mais tardios descritos, com início dos sintomas em até 40 dias. $^{3}$

O tratamento conservador da SAMS consiste na descompressão nasogástrica associada ao suporte nutricional e a correção dos distúrbios hidroeletrolíticos. Quando a drenagem através da sonda diminui para menos de $100 \mathrm{ml}$ em 8 horas, pode-se iniciar a ingestão de líquidos orais e avançar lentamente para alimentos pastosos em pequenas quantidades e com maior frequência..,

Grande parte dos pacientes que desenvolvem a SAMS evoluem de forma satisfatória com o tratamento conservador. Geralmente, as crianças têm evolução mais favorável do que os adultos, e os sintomas melhoram em torno de 2 a 3 dias de descompressão gástrica através da sondagem. ${ }^{5,6}$ Se os sintomas persistirem, o tratamento cirúrgico deve ser considerado, sendo as principais opções a gastrojejunostomia ou a duodenojejunostomia.

A SAMS pode ocorrer tanto nas correções cirúrgicas das cifoescolioses quanto durante o tratamento conservador das deformidades com colete de gesso, sendo conhecida também como "síndrome do gesso." A explicação para que a imobilização com colete inicie a compressão vascular do duodeno é 


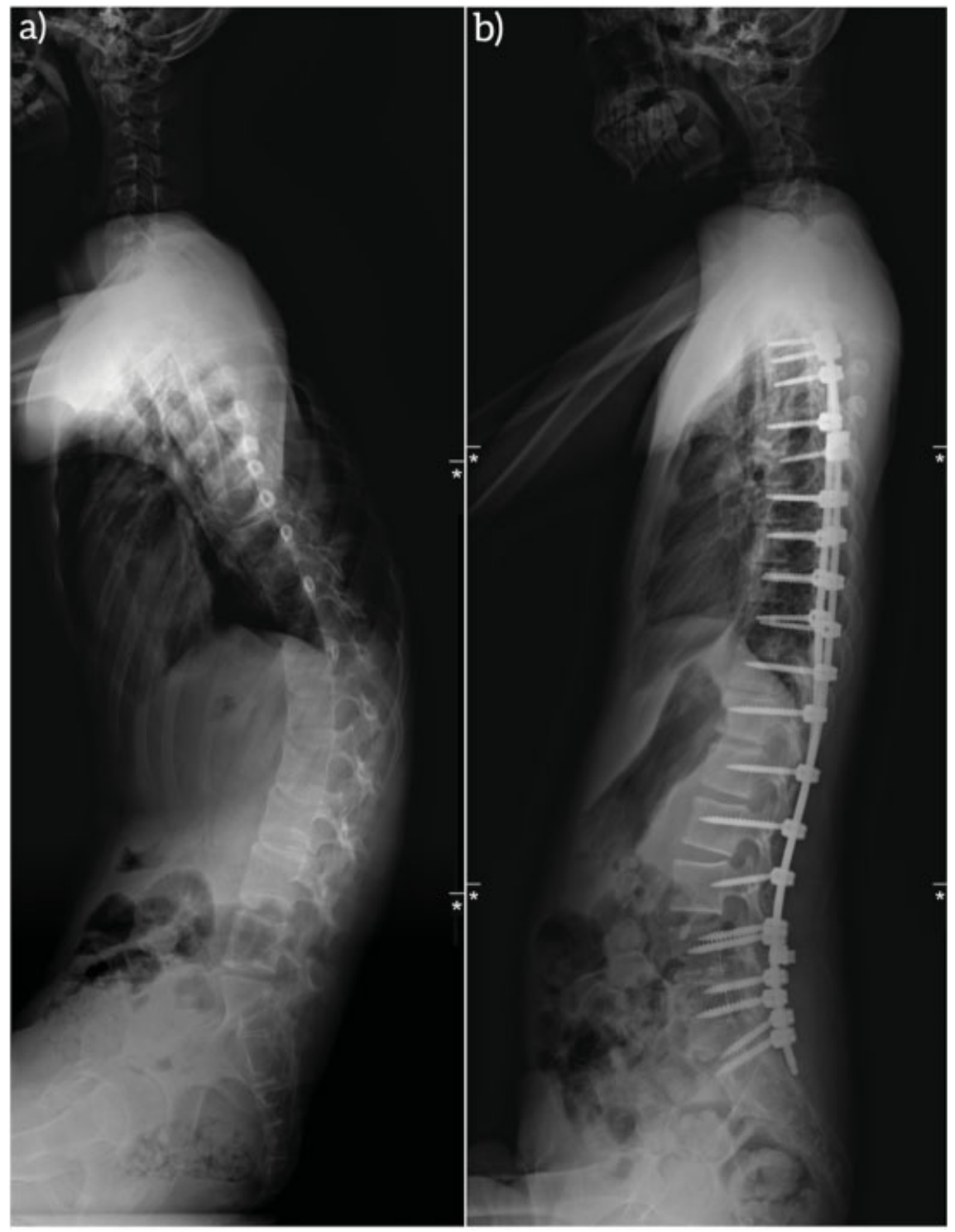

Fig. 3 (a) Radiografia panorâmica da coluna em perfil pré-operatória (b) Radiografia no pós-operatório.

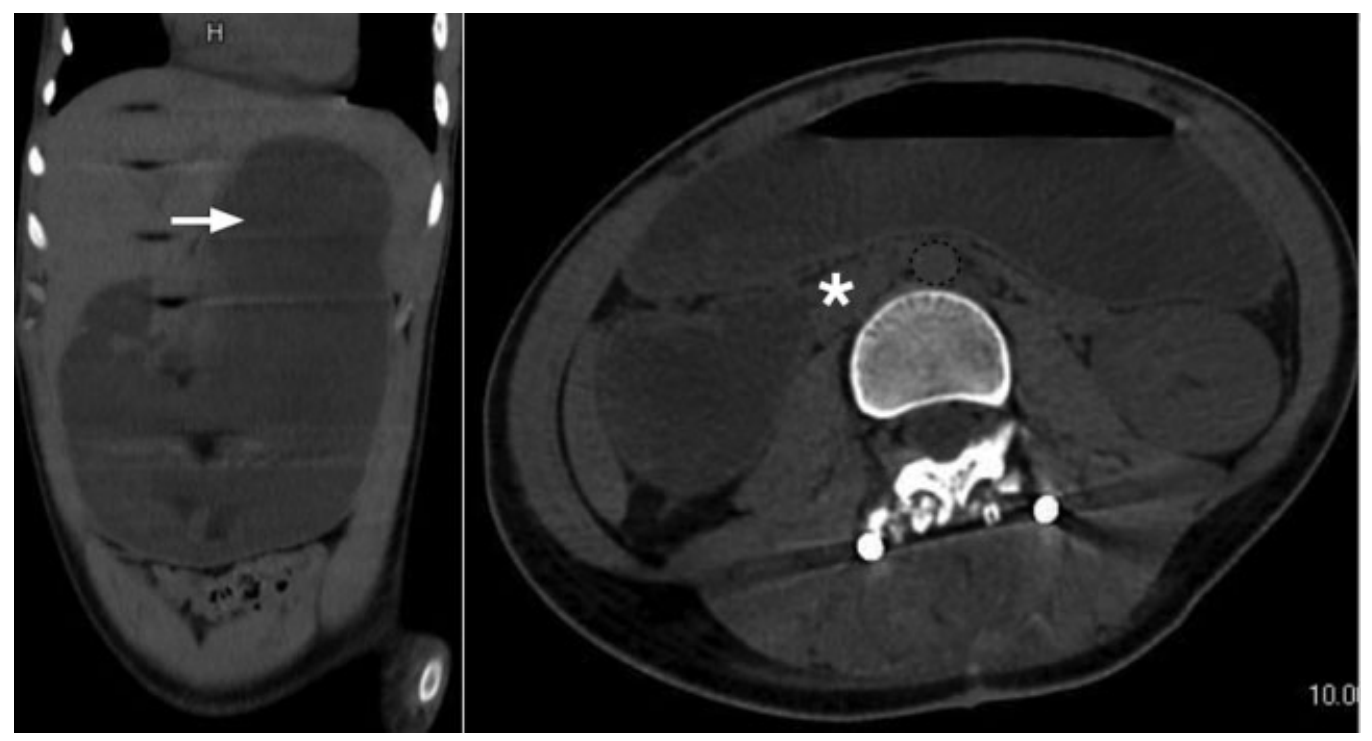

Fig. 4 Cortes tomográficos coronal e axial do abdome. (seta branca - distensão gástrica; ${ }^{*}$ afilamento do duodeno na $3^{\mathrm{a}}$ porção). 


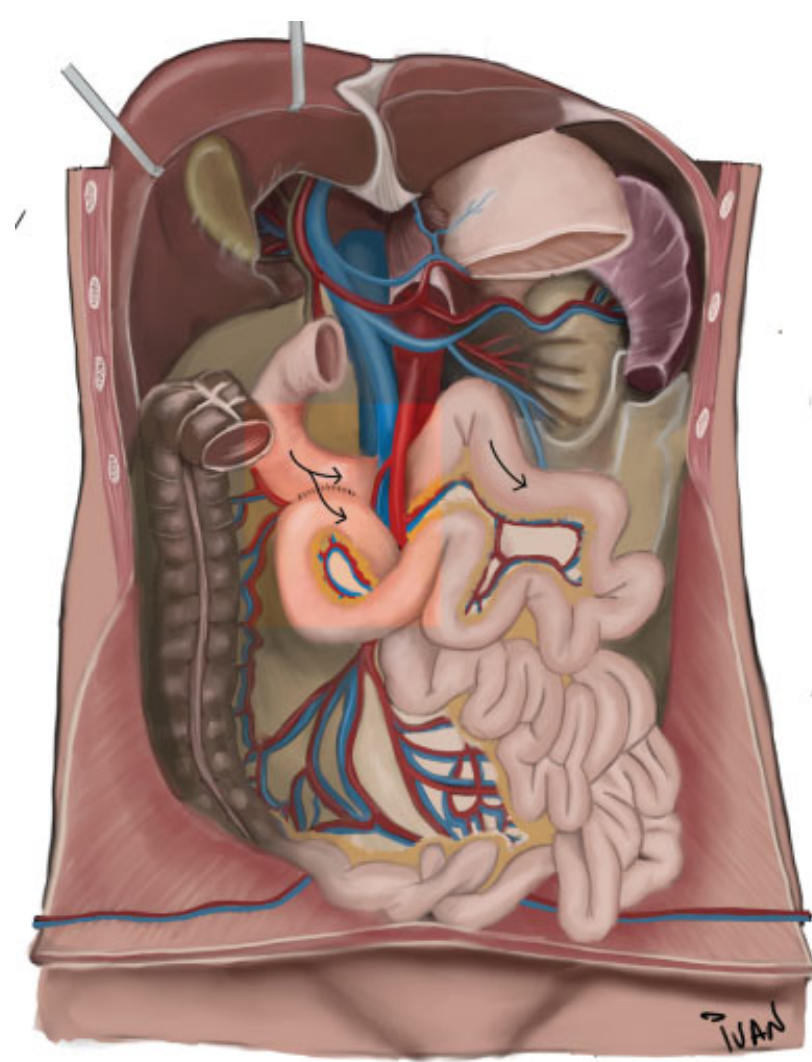

Fig. 5 Ilustração demonstrando a derivação duodenojejunal com redirecionamento do trânsito intestinal.

obscura. O primeiro caso de compressão vascular mesentérica associada à aplicação de colete gessado foi descrito por Willett em 1878, com o uso de um gesso de "Sayre" em um garoto de 17 anos, e a partir de então novos casos foram relatados com diferentes patologias geradoras de deformidades na coluna. Evarts et al. $^{9}$ descreveram 18 casos de pacientes que desenvolveram a síndrome no curso do tratamento de deformidades da coluna. Os autores alertaram para a necessidade de suspeitar de SAMS em pacientes sintomáticos após correções com colete gessado, coletes tipo Milwaukee, tração halofemoral ou correção cirúrgica. ${ }^{4,9-11}$

Altiok et al. realizaram um estudo retrospectivo no qual foram revisados 2.939 prontuários, sendo encontrados 17 casos de SAMS. Os autores avaliaram a influência das manobras de translação e derrotação vertebral na incidência da síndrome, e concluíram que as novas manobras de correção não eliminaram a síndrome. A própria correção da coluna leva ao seu alongamento, o que acarreta uma mudança no ambiente anatômico da artéria mesentérica superior. ${ }^{4}$

Braun et al. ${ }^{12}$ observaram que os pacientes com IMC menor que o $25^{\circ}$ percentil para a idade, aqueles com uma curva torácica mais rígida ( $<60 \%$ de correção nas radiografias de inclinação) e aqueles com uma curva lombar deslocada lateralmente (modificador lombar da classificação de Lenke tipo B ou C) estão em maior risco para o desenvolvimento da SAMS após a correção da deformidade. ${ }^{12,13}$ Carelli et al. ${ }^{11}$ relataram 2 casos de síndrome da artéria mesentérica superior ocorridos após correções de deformidades sagitais da coluna, nos quais o tratamento conservador da oclusão intestinal foi satisfatório para a resolução dos quadros. Os autores acreditam que a incidência da síndrome deva ser mais comum do que o descrito, com grande parte dos casos apresentando uma resolução espontânea.

A SAMS, embora seja incomum, é uma complicação que pode ocorrer após correções de deformidades da coluna. É importante que a equipe envolvida no tratamento tenha conhecimento da síndrome e um alto grau de suspeição na presença de sintomas gastrointestinais, para evitar atraso no diagnóstico e tratamento, evitando desfechos desfavoráveis.

Nota

Trabalho realizado no departamento de Ortopedia da rede Sarah de Hospitais de Reabilitação, Brasília, DF, Brasil.

\section{Suporte Financeiro}

Não houve suporte financeiro de fontes públicas, comerciais, ou sem fins lucrativos.

Conflito de Interesses

Os autores declaram não haver conflito de interesses.

\section{Referências}

1 Tsirikos AI, Anakwe RE, Baker AD. Late presentation of superior mesenteric artery syndrome following scoliosis surgery: a case report. J Med Case Reports 2008;2:9

2 Rabinovitch J, Pines B, Felton M. Superior mesenteric artery syndrome. JAMA 1962;179:257-263

3 Crowther MA, Webb PJ, Eyre-Brook IA. Superior mesenteric artery syndrome following surgery for scoliosis. Spine (Phila Pa 1976) 2002;27(24):E528-E533

4 Altiok H, Lubicky JP, DeWald CJ, Herman JE. The superior mesenteric artery syndrome in patients with spinal deformity. Spine (Phila Pa 1976) 2005;30(19):2164-2170

5 Voleti SPR, Sridhar J. Superior Mesenteric Artery Syndrome after Kyphosis Correction - A Case Report. J Orthop Case Rep 2017;7 (05):67-70

6 Bernotavičius G, Saniukas K, Karmonaitė I, Zagorskis R. Superior mesenteric artery syndrome. Acta Med Litu 2016;23(03):155-164

7 Jalanko T, Helenius I, Pakarinen M, Koivusalo A. Gastrointestinal Complications After Surgical Correction of Neuromuscular Scoliosis: A Retrospective Cohort Study. Scand J Surg 2018;107(03): 252-259

8 Tsirikos AI, Jeans LA. Superior mesenteric artery syndrome in children and adolescents with spine deformities undergoing corrective surgery. J Spinal Disord Tech 2005;18(03):263-271

9 Evarts CM, Winter RB, Hall JE. Vascular compression of the duodenum associated with the treatment of scoliosis. Review of the literature and report of eighteen cases. J Bone Joint Surg Am 1971;53(03):431-444

10 Zhu ZZ, Qiu Y. Superior mesenteric artery syndrome following scoliosis surgery: its risk indicators and treatment strategy. World J Gastroenterol 2005;11(21):3307-3310

11 Carelli LE, Schettino LC, Pedras CV, Pedras SV. Síndrome da artéria mesentérica superior após correção de deformidades vertebrais. Orthop Trauma 2004;1:5-10

12 Braun SV, Hedden DM, Howard AW. Superior mesenteric artery syndrome following spinal deformity correction. J Bone Joint Surg Am 2006;88(10):2252-2257

13 Lenke LG, Edwards CC 2nd, Bridwell KH. The Lenke classification of adolescent idiopathic scoliosis: how it organizes curve patterns as a template to perform selective fusions of the spine. Spine (Phila Pa 1976) 2003;28(20):S199-S207 